\title{
Structural and Elastodynamic Analysis of Rotary Transfer Machines by Finite Element Model
}

\author{
A. Martini ${ }^{1,2^{*}}$, M. Troncossi ${ }^{1,2}$, N. Vincenzi ${ }^{3}$ \\ ${ }^{1}$ DIN - Dept. of Engineering for Industry, University of Bologna, Viale del Risorgimento 2, \\ 40136 Bologna, Italy \\ alberto.martini6@unibo.it \\ marco.troncossi@unibo.it \\ ${ }^{2}$ CIRI - Advanced Applications in Mechanical Engineering and Materials Technology, \\ University of Bologna, V.le del Risorgimento 2, 40136 Bologna, Italy \\ ${ }^{3}$ GIULIANI - a Bucci Automations SpA Division, Via Granarolo 167, 48018 Faenza, Italy \\ n.vincenzi@bucci-industries.com \\ *corresponding author
}

\begin{abstract}
Vibration monitoring and control are central topics for machine tools, since high vibration levels reduce the quality of machined surfaces and shorten the tool life. In order to predict potential vibration issues since the early design stage, it is necessary to implement ad hoc numerical models for modal analysis. This requires significant efforts and possible conflicts with tight production scheduling of companies. This work focuses on a specific family of rotary transfer machines for the manufacturing of parts related to lock\&keys industry. It investigates the possibility to achieve an acceptable estimation of the elastodynamic behavior of the machine tools through limited modifications of the Finite Element (FE) models used for structural analysis, which are generally available in the early phases of the design process. The structural FE model of a new machine tool is implemented and validated through experimental tests performed on a prototype. Then, the elastodynamic FE model is derived and simulated. The numerical results are consistent with the data provided by Experimental Modal Analysis (EMA). Hence, the proposed approach is confirmed viable and will be integrated in the company workflow of future machine designs.
\end{abstract}

Keywords: structural analysis, elastodynamic analysis, Experimental Modal Analysis, lock\&keys industry, rotary transfer machine

\section{Introduction}

High-performance machineries for industrial applications, such as high-speed automated assembly lines and machine tools, frequently experience vibrations induced by high dynamic loads, which are possibly detrimental for their operation (Altintas 2012, Liang et al. 2013, Martini et al. 2016, Fragassa 2017). In practice, due to company production scheduling and resource issues, vibration issues are often detected at late steps of the design workflow, when the machine prototype is assembled and firstly tested. In such an instance, experimental measurements are generally performed in order to identify the critical parts of the machine and 
properly modify their design (Schedlinski et al. 2002, Liang et al. 2013, Pavlovic et al. 2016). This process eventually proves costly and time consuming.

As for machine tools, there are several possible sources of vibrations. The most relevant ones are the cutting forces between tools and workpieces, which can excite the system resonances in a wide frequency band (primarily determined by the tooth passing frequencies and their harmonics) and induce chatter phenomena and high vibration levels (Altintas et al. 2004, Mahnama et al. 2012, Sam Paul et al. 2016, Zigulic et al. 2017). Additionally, inertial loads associated with rapid movements for tool positioning, or even vibrations transmitted by the ground, can trigger elastodynamic phenomena (Troncossi et al. 2009, Wójcicki et al. 2015). Chatter and severe vibrations of both the workpiece holder and the machining head degrade the quality of the machined surfaces, thus reducing the productivity, due to considerable amounts of nonconforming products. They also involve durability issues by reducing the tool life and increase wear of the components of the mechanical power transmission chain. Hence, predicting and solving potential vibration issues is essential to enhance the performance of machine tools and to limit the maintenance costs (Lucisano et al. 2016).

Despite the above considerations, the elastodynamic behavior of machineries remains not deeply investigated within the design process of many companies, because of two main factors. On the one hand, machineries designed according to strength criteria are typically verified by means of static structural analyses performed with extremely detailed and computationally demanding FE models (featuring very fine mesh and nonlinear modelling entities), in order to properly determine stresses and deformations and avoid failures of materials. Also, assessing the elastodynamic behavior of complex mechanical systems generally requires the implementation of FE models and, in practical applications, the use of FE models is advisable to properly estimate resonances and mode shapes of very simple mechanisms as well (e.g. Martini et al. 2013, Pavlovic et al. 2017). However, the models for structural analysis are not suitable to perform and integrate modal analysis within the CAE process, and developing new tailored FE models would be needed (thus requiring additional time and/or resources). On the other hand, numerous parameters (e.g. joint or bearing stiffness, material damping coefficients) must be properly set in FE models to achieve a sufficiently accurate prediction of the system elastodynamic behavior. Frequently these parameters are not available or not easily achievable (Nedic et al. 2017) so that the reliability of the analysis results could be weak. Hence, the investigation of vibration issues is generally postponed to the final design phase and performed by means of experimental tests on the machine prototype.

This work deals with the elastodynamic analysis of rotary transfer machines (Koch 2001, Landi et al. 2008), namely machine tools typically devised for the large-batch production of limited families of parts. This kind of machines is typically designed according to stiffness criteria (Stephenson et al. 2016). Accordingly, the FE models adopted for their structural analysis are generally implemented on the basis of simplified CAD geometries, by using relatively coarse mesh, since a detailed geometry description is not required (as opposite to stress analysis purposes, where features like notches, welding or small holes are fundamental). Hence, they are mostly compatible with the characteristics of FE models developed for modal analysis.

The main objective of the present study is investigating the possible use of elastodynamic FE models, directly derived from those adopted for structural stiffness analysis, to estimate natural frequencies and mode shapes with an acceptable accuracy (in the early stages of a new machine development, where high-level information and guidelines are required as design drivers, accuracy could be not very strict, e.g. errors up to $15-20 \%$ may be still acceptable to understand if certain modes can be excited during operations). This approach may permit to develop predictive tools (sufficiently reliable though not particularly accurate) to be integrated 
in the design process with very little efforts. In particular, the elastodynamic FE models, although not experimentally validated, may allow the identification of potential vibration issues and provide the guidelines to deals with them since the early phases of the design process.

This work focuses on a new 9-station rotary transfer machine for the manufacturing of lock components. Fortunately, a prototype of the studied machine is already available so that a validation of the models and the evaluation of the proposed procedure accuracy is possible. The static structural FE model is implemented and validated by experimental tests. The validated model is used as a starting point to derive another FE model suitable for modal analysis, with very limited modifications. EMA is performed on the prototype to confirm the viability of the proposed approach for this family of rotary transfer machines.

The paper is organized as follows. Section 2 presents in details the studied rotary transfer machine. Section 3 describes the validated FE model for structural analysis. Section 4 presents the implementation of the FE model to analyze the elastodynamic behavior of the machinery, and its experimental verification through EMA. The final Section provides the concluding remarks.

\section{Machinery description}

The system under investigation is designed to perform different machining and assembly operations in lock\&keys industry, such as the double cylinder body (Fig. 1a), at high production capacity (over 1 million parts/year). It features nine functional stations which are located on a fixed plate in the inner part of the machine (Fig. 1b): the first one is devised to the automatic loading and unloading of the workpieces; the other eight stations include multi-spindle CNC machining units as well as automated feeders and manipulating units to orient the workpieces and assemble additional components. A rotary indexing table with vertical axis is composed of both a large rotary plate (diameter almost $1700 \mathrm{~mm}$ ) and the attached clamping elements (namely the clamps and their cantilever supports) that hold the workpieces (Fig. 2).

(a)

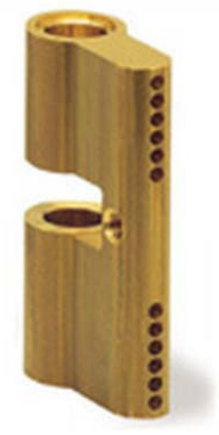

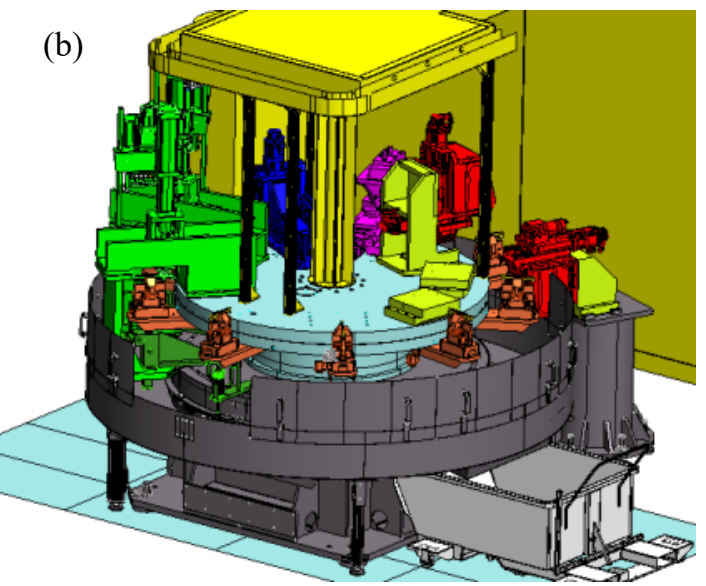

Fig. 1. (a) Machined lock part; (b) CAD model of the complete machinery

A torque motor directly drives the rotary plate and keeps it locked during the machining operations, without the need of further couplings and hydraulic pressures (e.g. Hirth ring indexing). The rotary table has been optimized to present a relatively low inertia, by means of lightweight materials (primarily aluminum and CFRP) and high-stiffness geometries. Thanks to 
these features, the rotation time between stations is significantly reduced, hence limiting the Non-Productive Time (with respect to a standard Hirth based rotary table it is possible to get reductions up to $-75 \%$ ).

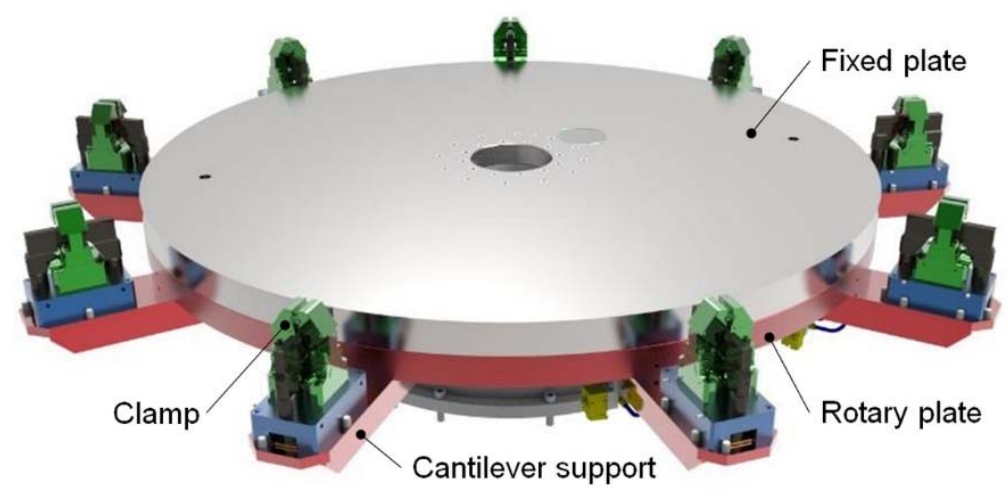

Fig. 2. Rendered CAD model of the rotary table with clamping elements

\section{Structural analysis of the rotary table}

Machine tools must be characterized by high stiffness in order to meet strict requirements in terms of production tolerances. The presence of static and/or dynamic loads with non-zero mean value, is a typical loading condition that characterizes most of the machining and assembly operations. Static analysis aims at assessing the machine structural stiffness by investigating the deformations induced by static loads on the machine structure and critical components (e.g. the workpiece clamp support). Elastodynamic analysis investigates the vibration behavior of the machine which could play a significant role from the accuracy perspective during operation. The former is a common practice in company technical offices and is usually adopted since the early stage of the machine design. The latter is generally intended as an advanced analysis addressed to critical systems, due to the greater complexity of the studies to perform and the corresponding time required (in possible contrast with time delivering issues), above all for the elastodynamic model validation by means of experimental data (hardly available). In this study the structural model used for static analysis is exploited as it is also for the elastodynamic investigation, limited to a modal analysis.

\subsection{Structural FE model}

The FE model is implemented starting from the 3D CAD model of the machine (Fig. 2). As usual, the CAD model is simplified by means of defeaturing tools. The rotary table is expected to be the most critical component. Hence, the structural analysis here presented focuses on the rotary table, whereas other components (e.g. the functional units) are not taken into account in the FE model.

Mirror structural symmetry with respect to vertical planes is exploited to mesh only a half of the machine structure. The fixed plate is meshed with 20 -node bricks and its base is rigidly constrained to the fixed ground, which is considered non-deformable. Tetrahedral elements with 10 nodes are used for both the rotary plate and the cantilever clamp supports, as they present some small geometries that cannot be omitted. The clamps are not included in the FE mesh, since they are expected to exhibit negligible deformations with respect to the other components of the rotary table. Nonetheless, the clamp geometry is taken into account to define the 
simulated loading conditions. In particular, the static loads are applied to a location that coincide with the median point of the clamp (i.e. where the workpiece is hold, Fig. 3a), which is rigidly constrained to the clamp bolt connections. In this preliminary model, the bearing of the rotary table (namely a crossed roller bearing with external diameter almost $800 \mathrm{~mm}$ ) is approximated through a steel ring. The connections of the bearing and of all the cantilever clamp supports are modelled as bonded constraints between the nodes of the mesh. The model has about $9.5 \cdot 10^{4}$ nodes, for a total amount of about $2.85 \cdot 10^{5}$ DOFs.

The effects of three different loading conditions are evaluated: (i) downward vertical load (Fig. 3a); (ii) inward radial load; (iii) outward radial load. For all the conditions, a constant magnitude of $1 \mathrm{kN}$ is considered. However, since the loads are applied to the halved cantilever, their magnitudes are halved as well in the simulations.

The deflection induced on the rotary table by the loads is computed through static structural simulations, starting with standard bonded contacts between all the parts of the table. The analysis focuses on the tip displacements of the cantilever clamp supports, in order to assess how the loads characterizing one functional station may affect the operations of the station itself as well as of the neighboring ones.

As expected, the simulation results show that the most critical case is associated with the vertical load condition (Fig. 3b). In particular, the point of application of the vertical load exhibits a downward displacement of about $0.1 \mathrm{~mm}$.

(a)

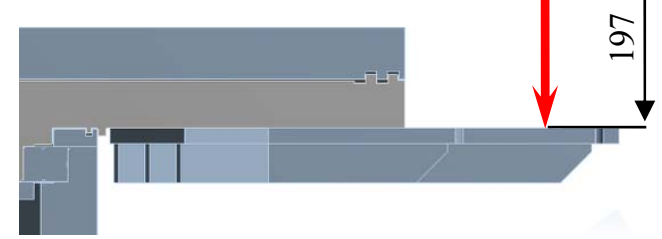

(b)

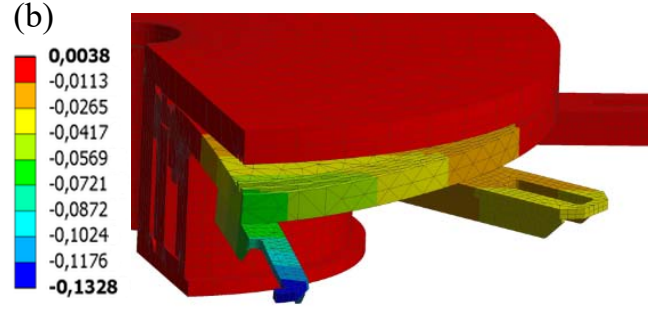

Fig. 3. (a) Vertical static load condition; (b) Rotary table deflection for the vertical load

\subsection{Model updating and validation}

Experimental tests are performed on the machine prototype to verify the deformations induced by a static vertical load. A constant vertical load of $1 \mathrm{kN}$ is applied to one of the cantilever clamp support by means of dead weights. The deflection is measured by using dial test indicators with resolution of $2 \mu \mathrm{m}$, in three relevant locations (Fig. 4): (i) projection of the point of application of the load on the cantilever clamp support (point $\mathrm{C} 1$ ); (ii) location homologous to $\mathrm{C} 1$ on the neighboring support (point $\mathrm{C} 2$ ); (iii) point of the loaded support close to its connection with the rotary plate (point $\mathrm{C} 3$ ).

Table 1 shows the comparison between the measured deflection $\left(\delta_{E X P}\right)$ and the vertical displacement computed through structural simulations $\left(\delta_{F E M}\right)$. The percentage error of the estimation provided by the FE model is computed as:

$$
\Delta \delta=\frac{\delta_{F E M}-\delta_{E X P}}{\delta_{E X P}} \cdot 100
$$


The computed deflections are significantly underestimated for all locations. Hence, the FE model appears too stiff, an updating of its parameters being thus required to match the available experimental data.
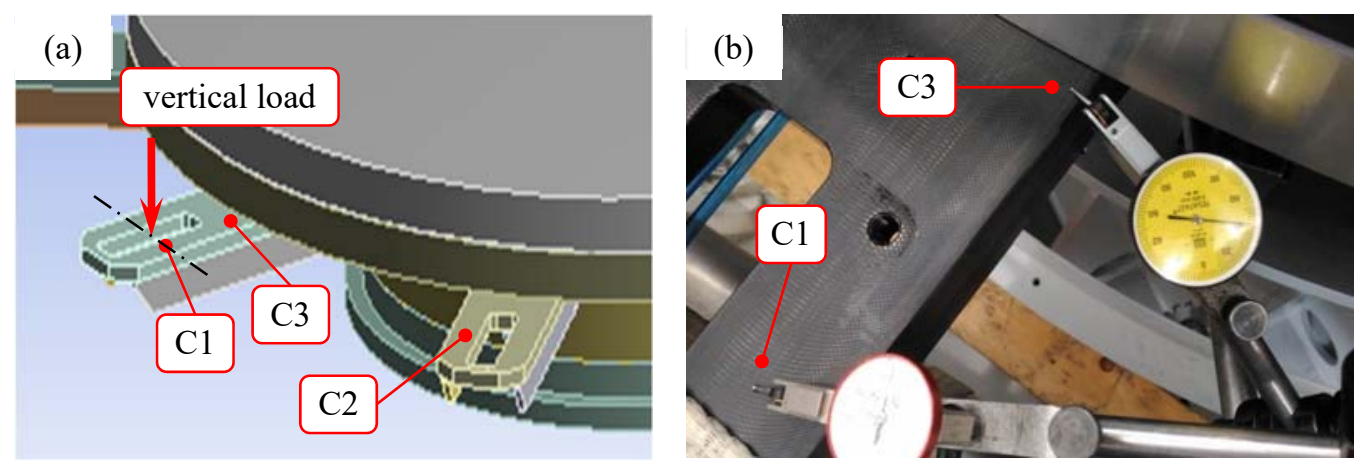

Fig. 4. (a) Monitored points; (b) Close-up of the dial test indicators

\begin{tabular}{cccc}
\hline Location & $\begin{array}{c}\boldsymbol{\delta}_{\text {FEM }} \\
{[\mathbf{m m}]}\end{array}$ & $\begin{array}{c}\boldsymbol{\delta}_{\text {EXP }} \\
{[\mathbf{m m}]}\end{array}$ & $\begin{array}{c}\Delta \boldsymbol{\delta} \\
{[\%]}\end{array}$ \\
\hline $\mathrm{C} 1$ & 0.100 & 0.120 & $-16.7 \%$ \\
\hline $\mathrm{C} 2$ & 0.025 & 0.040 & $-37.5 \%$ \\
\hline $\mathrm{C} 3$ & 0.060 & 0.080 & $-25.0 \%$ \\
\hline
\end{tabular}

Table 1. Comparison between measured and computed deflection with standard bonded contact between parts and rigid roller bearing

The model of the rotary table bearing is updated by taking into account the estimated equivalent stiffness of the crossed roller bearing and the effects of the bearing preload (according to the stiffness curves provided by the bearing manufacturer). The bonded constraints between the table and the cantilever clamp supports are replaced with models of the screw joints that characterize the actual design of the machine (namely, four metric screws M12 for each support). In particular, friction is introduced at the interface between each support and the rotary plate, as well as between the screw heads and each support (friction coefficient equal to 0.2 ); a tightening load of $70 \mathrm{kN}$ is applied for each M12 screw to take into account its preload.

The comparison between experimental data and the new results, obtained after model updating, is reported in Table 2. The updated FE model provides a quite accurate estimation of the deflection of all the monitored locations. The new results appear sufficiently satisfactory, with maximum errors decreasing from $20 \mu \mathrm{m}$ to $4 \mu \mathrm{m}$. Hence, the FE model for structural analysis is considered validated and suitable for the elastodynamic analysis as well. 


\begin{tabular}{cccc}
\hline Location & $\begin{array}{c}\text { Updated } \boldsymbol{\delta}_{\mathrm{FEM}} \\
{[\mathbf{m m}]}\end{array}$ & $\begin{array}{c}\boldsymbol{\delta}_{\mathrm{EXP}} \\
{[\mathbf{m m}]}\end{array}$ & $\begin{array}{c}\Delta \boldsymbol{\delta} \\
{[\%]}\end{array}$ \\
\hline $\mathrm{C} 1$ & 0.124 & 0.120 & $+3.3 \%$ \\
\hline $\mathrm{C} 2$ & 0.037 & 0.040 & $-7.5 \%$ \\
\hline $\mathrm{C} 3$ & 0.083 & 0.080 & $+3.8 \%$ \\
\hline
\end{tabular}

Table 2. Comparison between measured and computed deflection after model updating

\section{Elastodynamic analysis of the rotary table}

Modal analysis provides the system modal parameters, which are essential to predict the elastodynamic response of the machinery under the effects of transient loads. This study aims at implementing an FE model for estimating modal parameters with a satisfactory reliability, by making only limited modifications of the model for static structural analysis (described in Section 3), with the idea that such approximation and simplicity is appreciated by companies from the production workflow viewpoint.

\subsection{Elastodynamic FE analysis}

The rotary table is expected to exhibit more severe vibrations, since its resonances can be excited by both the dynamic loads associated with the machining/assembly operations (namely cutting forces and insertion forces, respectively) and the inertial loads induced by the table rapid motion. Hence, elastodynamic analysis focuses on the rotary table.

In order to properly estimate the mode shapes and natural frequencies, it is not possible to consider only half model. Instead, a cyclic symmetry should be exploited (Mac Donald 2007, Livings et al. 2012). However, the validated model will be also used to estimate the Frequency Response Functions (FRFs) of the machinery through harmonic analysis, by applying a harmonic force to one single clamp (a loading condition that do not satisfy the cyclic constraint). Hence, the entire rotary plate with all the cantilever clamp supports are meshed. The same mesh pattern and element type of the model for static structural analysis are adopted. The FE model includes the bearing and the fixed plate (whose base is rigidly connected with the ground). The same constraints are also kept, except for the connections of the cantilever clamp supports. Indeed, the non-linear friction model is not supported in modal analysis (which is solved through linearization of the system). Hence, bonded joints are restored, but the normal stiffness parameter is reduced by two orders of magnitude with respect to the default value, in order to get the same deflection of the screw connection.

The clamps are not meshed as three-dimensional components, since their deformations are considered negligible. Conversely, their inertial contributions are deemed relevant for the elastodynamic behavior of the table, since each clamp is about $12 \mathrm{~kg}$. The mass and inertia of each clamp are taken into account in the FE model by means of a point mass remotely constrained to the corresponding cantilever clamp support.

Modal analysis is performed in the frequency range $0-400 \mathrm{~Hz}$, the tooth passing frequencies of the machining operations being between 100 and $300 \mathrm{~Hz}$. Indeed, even if the harmonics of the tooth passing frequencies may excite a much wider frequency band, the amplitude of vibrations for higher modes are reasonably expected to be extremely lower than for the first resonances. 
Table 3 shows the natural frequencies computed in the band of interest and a description of the corresponding mode shapes. A representation of the mode shapes is also reported in Fig. 5. Except for modes \#6 and \#7 (which exhibit a local torsion of the cantilever clamp supports whereas the rotary plate remains basically not deformed), the rotary table behaves very similarly to a simple annular plate clamped at its internal radius (Leissa 1969). Consequently, the modes are described by using the same convention, where $n$ denotes the number of nodal diameters and $s$ the number of nodal circles, not including the clamped circle (i.e. the inner boundary constrained to the bearing).
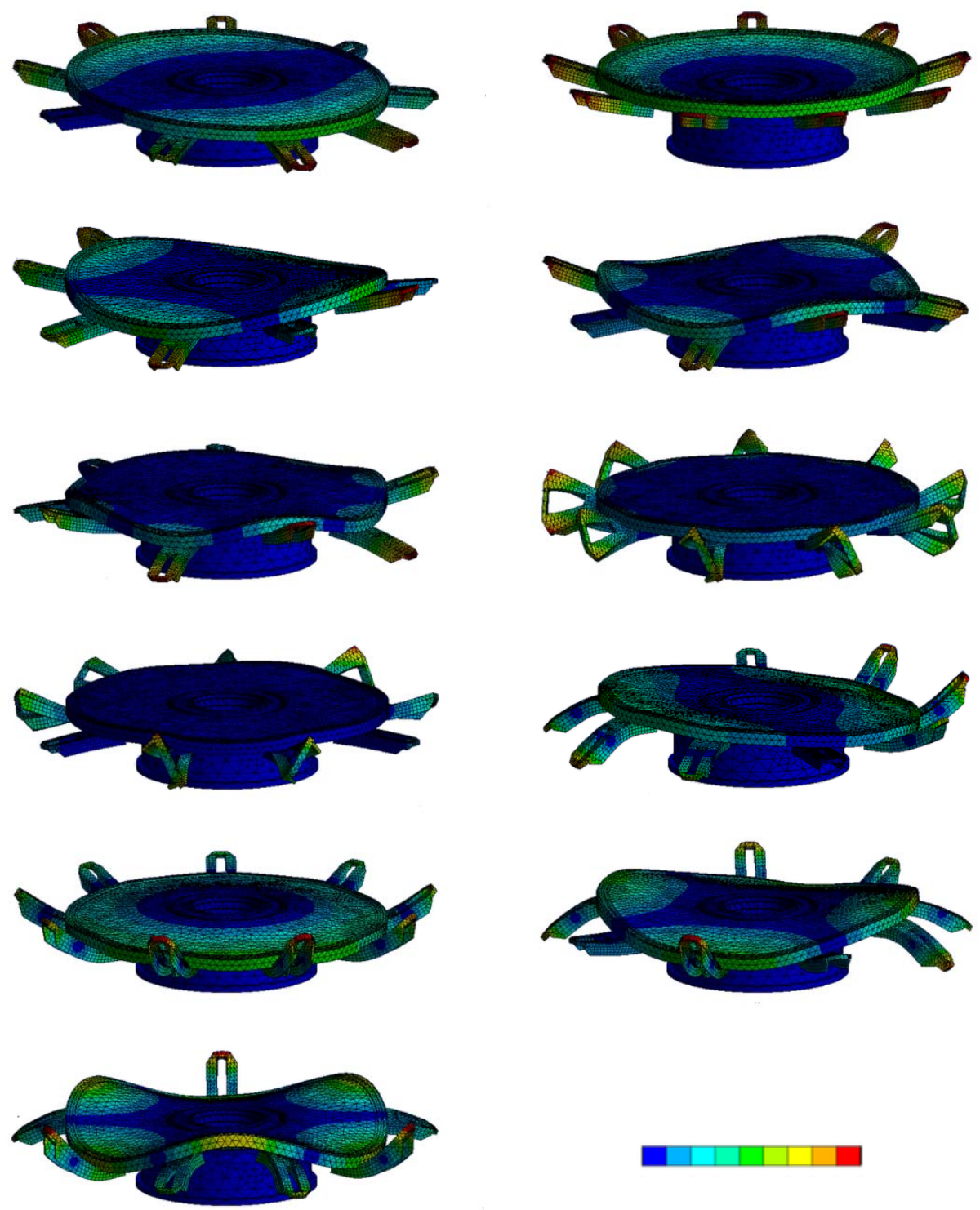

Fig. 5. Mode shapes of the rotary table computed thorough FEM (fixed plate not shown) 


\begin{tabular}{ccc}
\hline $\begin{array}{c}\text { FEM } \\
\text { mode no. }\end{array}$ & $\begin{array}{c}\boldsymbol{f}_{\boldsymbol{n}} \\
{[\mathbf{H z}]}\end{array}$ & mode shape description \\
\hline 1 & 80.7 & plate mode $\mathrm{n}=1, \mathrm{~s}=0$ \\
\hline 2 & 81.7 & plate mode $\mathrm{n}=0, \mathrm{~s}=0$ \\
\hline 3 & 89.1 & plate mode $\mathrm{n}=2, \mathrm{~s}=0$ \\
\hline 4 & 117.9 & plate mode $\mathrm{n}=3, \mathrm{~s}=0$ \\
\hline 5 & 143.7 & plate mode $\mathrm{n}=4, \mathrm{~s}=0$ \\
\hline 6 & 198.6 & local clamp torsion $(\mathrm{phased})$ \\
\hline 7 & 209.5 & local clamp torsion $($ counter-phased $)$ \\
\hline 8 & 307.1 & plate mode $\mathrm{n}=1, \mathrm{~s}=1$ \\
\hline 9 & 307.7 & plate mode $\mathrm{n}=0, \mathrm{~s}=1$ \\
\hline 10 & 314.4 & plate mode $\mathrm{n}=2, \mathrm{~s}=1$ \\
\hline \multirow{2}{*}{31} & 345.5 & plate mode $\mathrm{n}=3, \mathrm{~s}=1$ \\
\hline
\end{tabular}

Table 3. Computed natural frequencies and mode shapes up to $400 \mathrm{~Hz}$

Unexpectedly, the main contribution to the deformations of the lowest mode shapes, particularly the first three modes, is given by the rotary plate. Conversely, the cantilever clamp supports undergo small local deformations and rigidly follow the oscillations of their attachments to the rotary plate.

\subsection{EMA analysis and comparison}

The software package LMS Test.Lab is used for the experimental tests. A grid of points is firstly defined to simply represent the machine geometry in EMA. The grid selected for this application has 16 master points, which span a circular sector of $240^{\circ}$ thus including six of the nine clamps (Fig. 6): points $M_{i}(i=1, ., 6)$ are associated with one external corner of each cantilever clamp support; points $P_{j}(j=0, . ., 6)$ are placed on the external radius of the rotary plate, at equal distance from the clamps. This choice is made on the basis of the FEM results and permits to limit the number of the geometry size by exploiting the symmetry of the estimated mode shapes. Three additional points (namely $M_{5 s}, M_{5 t}$ and $M_{5 b}$ ) are located on the fifth clamp in order to possibly detect its torsional mode shapes. Further slave points (not highlighted with green markers in Fig. 6), defined by linear interpolation of two adjacent master points $P_{j}$, are also added to enhance mode shape animations thus helping to achieve a better interpretation of EMA results.

In EMA data processing the concept of geometry point turns into "node", namely a point plus one up to three directions required to univocally define both the locations and the directions of the input and the response measurements. This is needed to estimate the experimental FRFs matrices used to extract the modal parameters (natural frequencies, damping 
factors, and mode shapes). How many nodes are derived from a given set of geometry points depends on both the used sensors (measuring the responses) and the number of input locations and directions. In this application, piezoelectric accelerometers are installed in each master point of the grid (Fig. 7), except for points $M_{5 t}$ and $M_{5 b}$. In particular, a triaxial transducers is placed in $M_{5}$ to monitor vertical, radial and tangential vibrations. Monoaxial transducers detect vertical accelerations in the other thirteen master points. An additional sensor measures the tangential acceleration of the plate in $P_{5}$. An impact hammer (PCB 086D05) is used for exciting the system (Fig. 8) according to a roving hammer approach. All the measuring nodes are excited with an impact force, except for the tangential direction of $P_{5}$ that was not physically possible. Points $M_{5 t}$ and $M_{5 b}$ are also included in the input node set to possibly excite the clamp torsional modes. Hence, a total amount of 17 measuring nodes and 19 input nodes can be used to estimate the FRFs matrices. The resulting set up and test procedure appear complex and intricate, by combining the "roving hammer" and "roving accelerometer" approaches. This is done on purpose in order to analyze the acquired data, highly redundant, according to different processing based on different FRFs matrices (estimated from diverse subsets of input/response measurements) in order to find the most effective one.
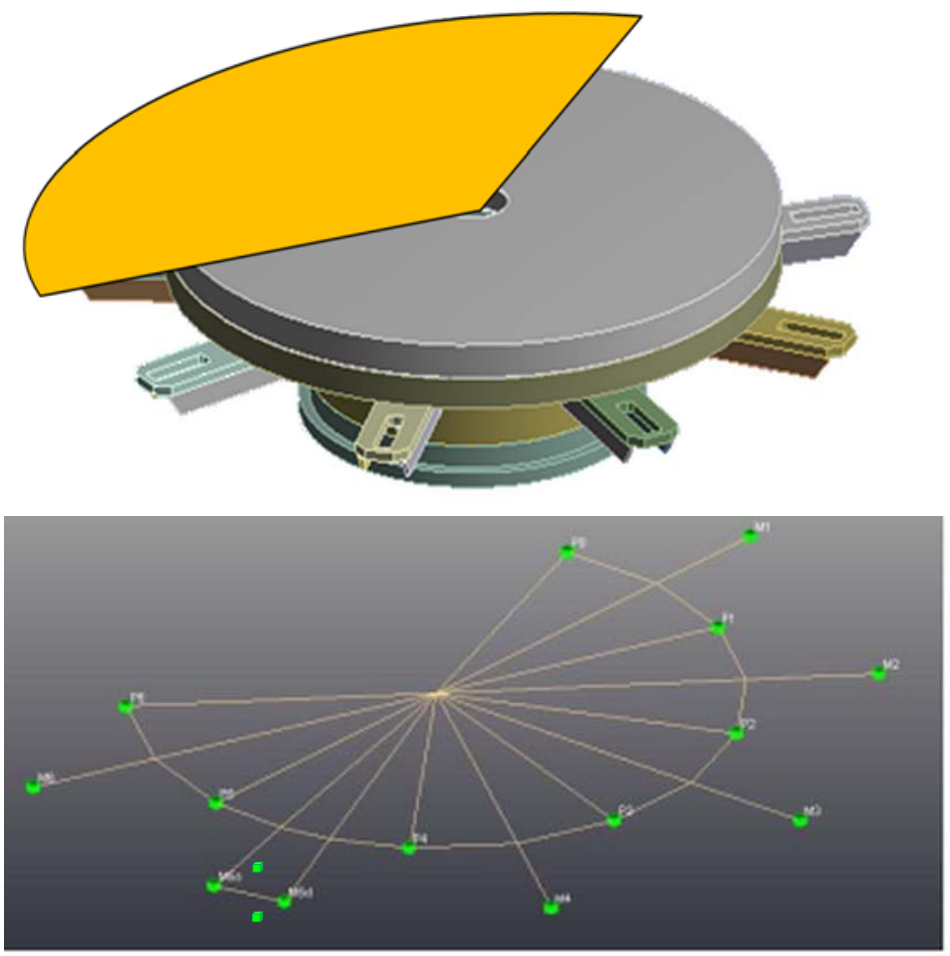

Fig. 6. Grid of points for EMA tests

Acquisitions are performed by using a LMS SCADAS SCM-05, with the following acquisition parameters: sampling frequency $\mathrm{Fs}=1024 \mathrm{~Hz}$; acquisition time $\mathrm{T}=5 ; 5$ averages for each measurement.

The system resonances are found by manually selecting the stable poles of the computed FRFs in the stabilization diagram (Fig. 9), which is obtained with PolyMAX algorithm (Peeters et al. 2004). Then the mode shapes are synthesized and validated by means of three indicators, 
namely Modal Phase Collinearity (MPC), Mean Phase Deviation (MPD), and Modal Participation (MP). The first indicator checks the degree of complexity of a mode (Juang et al. 1985). Real normal modes are characterized by a MPC index near $100 \%$ and values smaller than $90 \%$ generally indicate high damping or errors in the measurements and/or the analysis procedure. MPD (measured in degrees) indicates the phase scatter of a mode shape and should be very close to $0^{\circ}$ for real normal modes (Heylen et al. 1998), being values smaller than $15^{\circ}$ generally considered adequate for acceptance of results. The MP indicates how much a mode shape participates to the total system response. A low percentage value means that the mode is scarcely excited by the input forces.

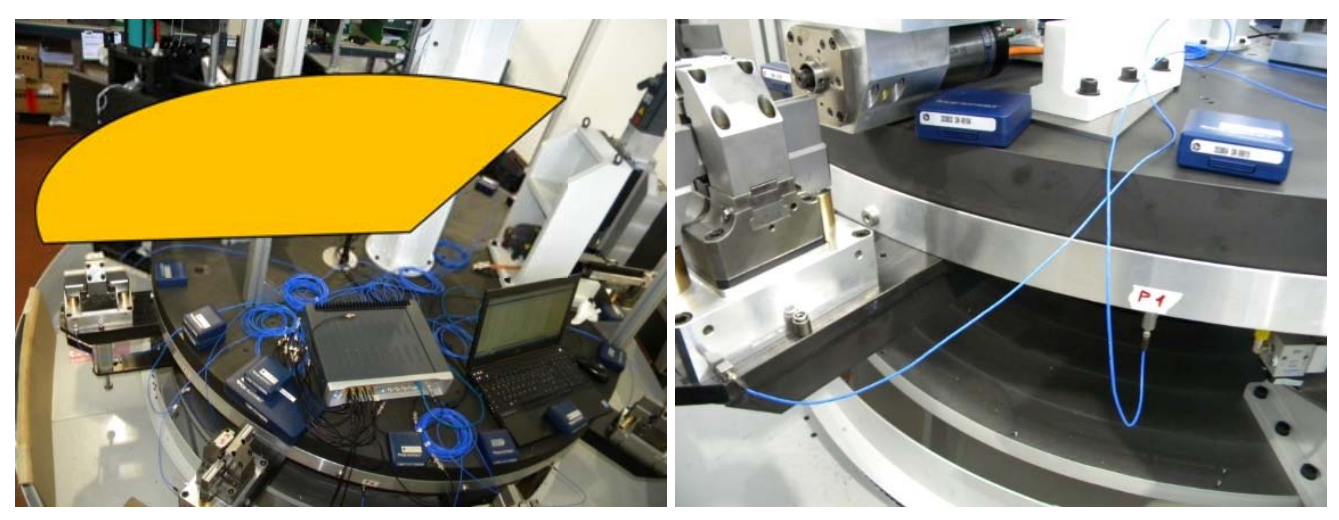

Fig. 7. Sensor setup for EMA tests

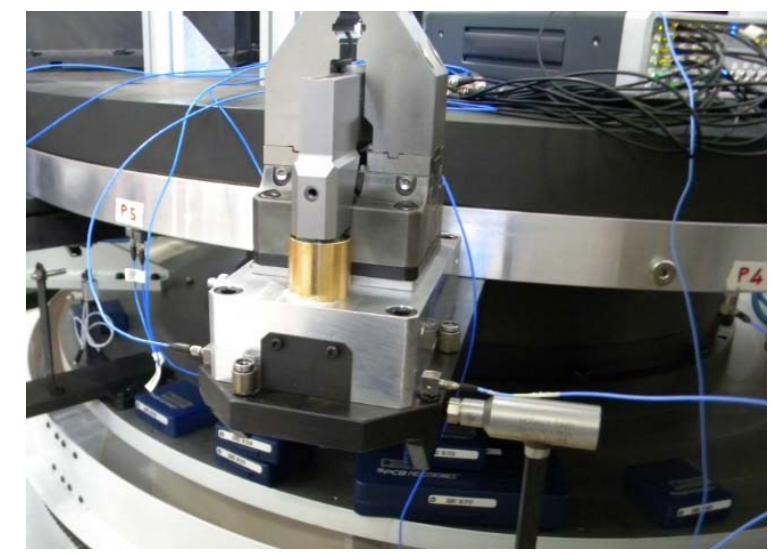

Fig. 8. Close-up of tangential impact excitation on clamp M5

Several combinations of input/measuring nodes (i.e. different FRFs matrices) has been tested in data analysis. The most accurate estimation of the modal parameters is achieved by considering the full FRF matrix, which includes all the 19 inputs and 17 outputs. This is quite unexpected since, in general, the best results are obtained by using relatively small subsets of the acquired signals. Indeed, measurements are usually affected by noise, which may cause overestimation of damping coefficients and poor reconstruction of the mode shapes, in case large datasets are used. Reasonably, data collected in this study are affected by negligible noise, so that the use of the full dataset provides a better estimation of the overall elastodynamic behavior of the machine. 
The results provided by the complete dataset are reported in Table 4 with the indicators adopted for assessing their reliability. Only five natural frequencies are found in the investigated frequency band $(0-512 \mathrm{~Hz})$. A representation of the corresponding mode shapes is shown in Fig. 10. The values of MPC and MPD indicate that the computed mode shapes are consistent with real normal modes, hence confirming that the estimate is sufficiently reliable. A local torsional mode of the clamping element defined by nodes $M_{5}$ appeared in some data processing, with inconsistent results depending on the FRFs set used, e.g. the natural frequency estimated $170 \mathrm{~Hz}$ in one case and $190 \mathrm{~Hz}$ in another one. Such uncertainty was confirmed by generally weak values of MPC and MPD indices associated to the mode. The MP index was always very low, thus meaning that the torsional mode was always scarcely excited (even when impacting the very clamp with the hammer). Due to the unreliable results and the low MP value, this local mode was not considered among the final results of the EMA.

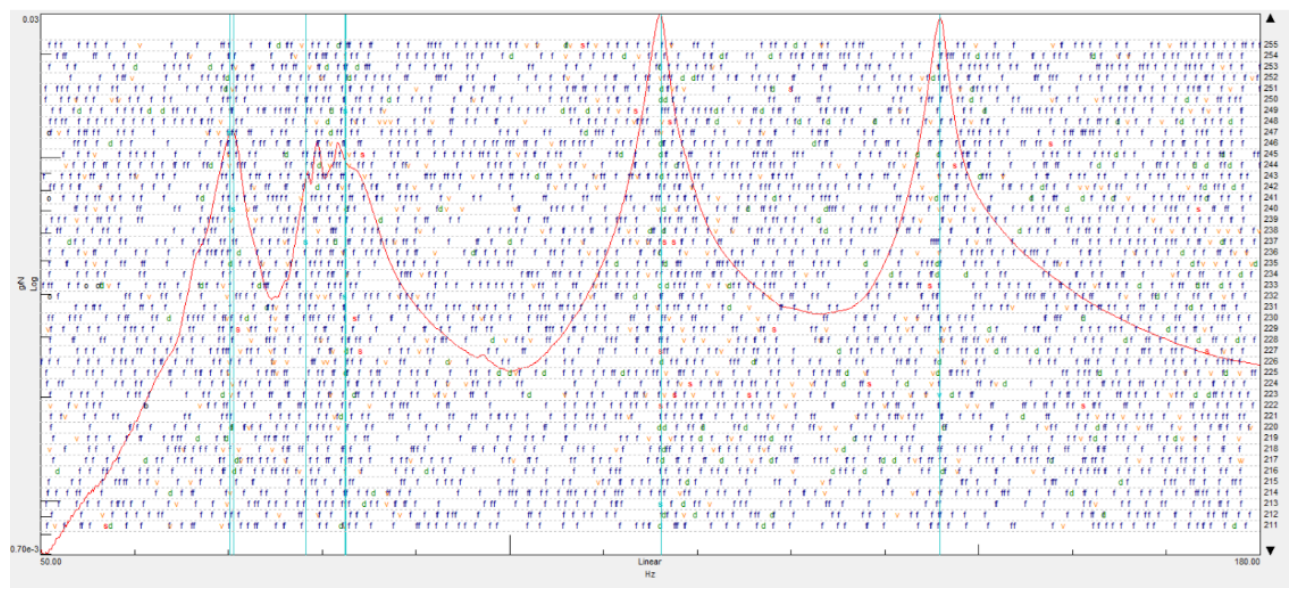

Fig. 9. Stabilization diagram with PolyMAX algorithm

The EMA results show that the first resonance of the analyzed rotary table is related to the mode shape without nodal diameters and nodal circles, i.e. $\mathrm{n}=0, \mathrm{~s}=0$ (Fig. 10), which is not in agreement with the results provided by the FE model (Table 3, Fig. 9). The first mode is also characterized by a rather low MP value, which indicates that it is scarcely excited by the impact hammer. From a practical viewpoint, it is worth noting that phased loads acting simultaneously on the clamps during machine operation may excite this mode. However, such loading condition is unlikely to occur in rotary transfer machines, since in general all the operations have very different characteristics with no predetermined synchronous timing. Therefore, this mode should not determine severe vibrations in the mechanical system under investigation. 


\begin{tabular}{cccccc}
\hline $\begin{array}{c}\text { EMA } \\
\text { mode no. }\end{array}$ & $\begin{array}{c}\boldsymbol{f}_{\text {n_EMA }} \\
{[\mathbf{H z}]}\end{array}$ & $\begin{array}{c}\text { mode shape } \\
\text { description }\end{array}$ & $\begin{array}{c}\text { MPC } \\
{[\%]}\end{array}$ & $\begin{array}{c}\text { MPD } \\
{[\mathbf{d e g}]}\end{array}$ & $\begin{array}{c}\text { MP } \\
{[\%]}\end{array}$ \\
\hline 1 & 66.8 & $\mathrm{n}=0, \mathrm{~s}=0$ & 98.5 & 5.4 & 8.1 \\
\hline 2 & 71.0 & $\mathrm{n}=1, \mathrm{~s}=0$ & 97.5 & 9.8 & 28.2 \\
\hline 3 & 82.0 & $\mathrm{n}=2, \mathrm{~s}=0$ & 99.4 & 5.2 & 26.1 \\
\hline 4 & 116.1 & $\mathrm{n}=3, \mathrm{~s}=0$ & 99.3 & 4.7 & 20.3 \\
\hline 5 & 145.7 & $\mathrm{n}=4, \mathrm{~s}=0$ & 99.7 & 3.4 & 16.8 \\
\hline
\end{tabular}

Table 4. Natural frequencies and mode shapes estimated by EMA
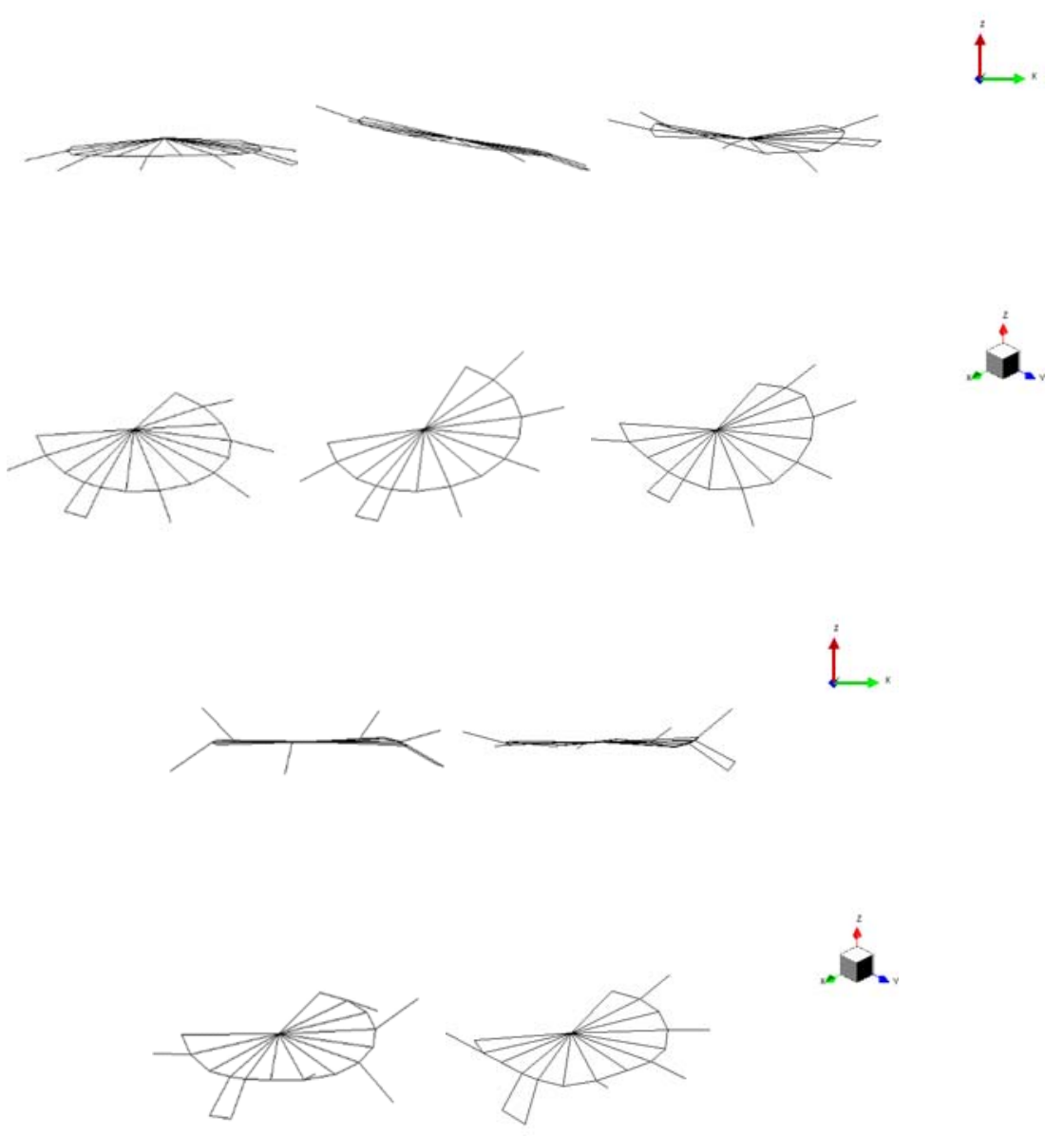

Fig. 10. Mode shapes of the rotary table estimated through EMA

Table 5 shows the comparison among measured and computed natural frequencies. The percentage error of the estimation provided by the FE model is computed as: 


$$
\Delta f_{n}=\frac{f_{n_{-} F E M}-f_{n_{-} E X P}}{f_{n_{-} E X P}} \cdot 100
$$

The second, third and fourth resonances are predicted quite well by the FEM. The first natural frequency is rather overestimated. However, since the corresponding mode shape without nodal diameters and nodal circles is reasonably not triggered in the actual operating condition of the machine, the approximation provided by the model is considered acceptable. As for the fifth natural frequency, an error below $15 \%$ is considered satisfactory as well. Indeed, the highest resonances normally exhibit smaller vibration amplitudes. Hence, they have a smaller influence on the system dynamic response, and a larger estimation error can be accepted.

The numerical and experimental mode shapes are also compared, by visual inspection. The results of FEM closely match the mode shapes estimated through EMA. In particular, the experimental data confirm that the deformations of the first three mode shapes are mainly ascribable to the vibrations of the rotary plate (Fig. 10).

In conclusion, the results of this study prove that an FE model directly derived from the one implemented for structural analysis permits to estimate the modal parameters of the rotary transfer machine under investigation with acceptable confidence. Hence, using this kind of numerical models appears a viable strategy to predict the elastodynamic behavior of the system, with acceptable accuracy, since the early phases of the design process, at least for machine tools with a similar architecture. In particular, this work provided valuable data concerning the modelling of the rotary table bearing and of the cantilever clamp supports. These data are currently being used to develop a new machinery belonging to the same family of the studied machine tool, namely a bigger rotary transfer machine with 15 functional stations.

\begin{tabular}{cccc}
\hline $\begin{array}{c}\text { EMA } \\
\text { mode no. }\end{array}$ & $\begin{array}{c}\boldsymbol{f}_{n_{-} E M A} \\
{[\mathbf{H z}]}\end{array}$ & $\begin{array}{c}\text { updated } \boldsymbol{f}_{-F E M} \\
{[\mathrm{~Hz}]}\end{array}$ & $\begin{array}{c}\Delta \boldsymbol{f}_{\boldsymbol{n}} \\
{[\%]}\end{array}$ \\
\hline 1 & 66.8 & 78.0 & +16.7 \\
\hline 2 & 71.0 & 76.9 & +8.3 \\
\hline 3 & 82.0 & 83.6 & +2.0 \\
\hline 4 & 116.1 & 106.8 & -8.0 \\
\hline 5 & 145.7 & 125.9 & -13.6 \\
\hline
\end{tabular}

Table 5. Comparison between measured and computed natural frequencies

\section{Conclusions}

This work studied the structural and elastodynamic behavior of a rotary transfer machine for lock\&keys industry components manufacturing. In particular, it investigated the possibility of adopting simplified FE models, directly derived from FE models for static stiffness analysis, to achieve an acceptable estimation of the machine modal parameters.

A FE model for stiffness analysis was implemented for the specific case of a rotary transfer machine for lock parts manufacturing, and validated through measurements on the machine tool prototype. Then, some minor modifications where made to obtain a model suitable for modal 
analysis. The computed modal parameters were compared with the results provided by EMA. Aiming at integrating numerical modal analysis in the early design stage of such a complex system, the numerical/experimental data matching can be considered acceptable being able to provide significant indications on the elastodynamic behavior and possible guidelines for design modifications in the case the dynamics is not acceptable. Hence, the viability of the proposed approach was confirmed, at least for the analyzed family of rotary transfer machines.

The study provided the practical guidelines to deal with some critical entities of the model, e.g. the clamp connections, in order to achieve a sufficiently satisfactory estimation of the elastodynamic behavior. The FE models developed in this way to be used in future machine developments, although not validated through experimental tests, are expected to provide essential data for an early assessment of potential vibration issues since the first steps of the design process. Indeed, this approach is going to be adopted by the manufacturer to drive the design of a rotary transfer machine of bigger size and similar architecture.

\section{Acknowledgements}

The study was conducted within the research project IGMI ECO-T, no. E28C15000190007, financed by the Operational Programme of the European Regional Development Fund of the Emilia-Romagna Region (ROP-ERDF 2014-2020, DGR 773/2015).

\section{References}

Altintas Y, Weck M (2004). Chatter stability of metal cutting and grinding, CIRP Annals Manufacturing Technology, 53 (2), 619-642.

Altintas Y (2012). Manufacturing Automation: Metal Cutting Mechanics, Machine Tool Vibrations, and CNC Design, Cambridge University Press.

Fragassa C (2017). Material selection in machine design: the change of cast iron for improving the high-quality in woodworking. Proceedings of the Institution of Mechanical Engineers, Part C: Journal of Mechanical Engineering Science, 231 (1), 18-30.

Heylen W, Lammens S, Sas P (1998). Modal Analysis Theory and Testing, KU Leuven, Leuven, Belgium, ISBN 90-73802-61-X.

Juang J-N, Pappa RS (1985). An eigensystem realization algorithm for modal parameter identification and model reduction. Journal of Guidance, Control and Dynamics, 8(5), 620627.

Koch LL (2001). Rotary transfer machine, Patent US6178608B1.

Landi E, Cusumano D (2008). Rotary transfer machine-tool with automatic tool change, Patent EP1985410A1.

Leissa AW (1969). Vibration of plates, NASA, Washington, D. C., US.

Liang Y, Chen W, Bai Q, Sun Y, Chen G, Zhang Q, Sun Y (2013). Design and dynamic optimization of an ultraprecision diamond fly cutting machine tool for large KDP crystal machining, International Journal of Advanced Manufacturing Technology, 69(1), 237-244.

Livings RA, Dayal V, Barnard DJ, Hsu DK (2012). Limitations of symmetry in FE modeling: A comparison of fem and air-coupled resonance imaging. Proc. AIP Conference 1430(31), 1816-1823. DOI: $10.1063 / 1.4716432$.

Lucisano G, Stefanovic M, Fragassa C (2016). Advanced Design Solutions for High-Precision Wood-working Machines. International Journal of Quality Research, 10 (1), 143-158.

Mac Donald BJ (2007). Practical stress analysis with finite elements, Glasnevin Publishing, Dublin, Ireland. 
Mahnama M, Movahhedy MR (2012). Application of FEM simulation of chip formation to stability analysis in orthogonal cutting process, Journal of Manufacturing Processes, 14, 188-194.

Martini A, Troncossi M, Rivola A (2013). Elastodynamic effects of mass-balancing: experimental investigation of a four-bar linkage, Advances in Mechanical Engineering, 2013, 949457, 1-10. DOI: 10.1155/2013/949457.

Martini A, Troncossi M (2016). Upgrade of an automated line for plastic cap manufacture based on experimental vibration analysis, Case Studies in Mechanical Systems and Signal Processing, 3, 28-33. DOI: 10.1016/j.csmssp.2016.03.002.

Nedić N, Dragan P, Fragassa C, Stojanović V, Pavlovic A (2017). Simulation of Hydraulic Check Valve for Forestry Equipment. International Journal of Heavy Vehicle Systems, 24 (3), 260-276 DOI: 10.1504/IJHVS.10001493.

Pavlovic A, Fragassa C, Ubertini F, Martini A (2016). Modal analysis and stiffness optimization: the case of ceramic tile finishing, Journal of the Serbian Society for Computational Mechanics, 10 (2), 30-44.

Pavlovic A, Fragassa C, Minak G (2017). Buckling Analysis of Telescopic Boom: Theoretical and Numerical Verification of Sliding Pads, Tehnicki Vjesnik, 24(3), 729-735, Doi: 10.17559/TV-20160510143822.

Peeters B, Van der Auweraer H, Guillaume P, Leuridan J (2004). The PolyMAX frequencydomain method: a new standard for modal parameter estimation? Shock and Vibration, 11(3-4), 395-409. DOI: 10.1155/2004/523692.

Sam Paul P, Raja P, Aruldhas P, Pringle S, Shaji E (2016). Effectiveness of particle and mass impact damping on tool vibration during hard turning process, Proceedings of the Institution of Mechanical Engineers, Part B: Journal of Engineering Manufacture, DOI: $10.1177 / 0954405416660995$.

Schedlinski C, Luscher M (2002). Application of Classical and Output-Only Modal Analysis to a Laser Cutting Machine, Proc. ISMA 2002, 16-18 September, Leuven, Belgium, 12731280.

Stephenson DA, Agapiou JS (2016). Metal Cutting Theory and Practice - Third Edition, CRC Press LLC, Boca Raton, FL, US. ISBN: 978-1-4665-8754-0.

Troncossi M, Troiani E, Rivola A (2009). Design optimization of a laser cutting machine by elastodynamic modeling, Proc. 9th Biennial Conference on Engineering Systems Design and Analysis (ESDA), Volume 2, 7-9 July 2008, Haifa, Israel, 551-559.

Wójcicki Z, Grosel J, Sawicki W, Majcher K, Pakos W (2015). Experimental (OMA) and numerical (FEM) modal analysis of ball mill foundations, Procedia Engineering, 111, 858863.

Zigulic R, Fragassa C, Skoblar A (2017). Influence of the longitudinal displacement on nonlinear principal parametric resonance of the woodworking bandsaw, Tehnicki Vjesnik 24 (1), 253-263. DOI:10.17559/TV-20160209175450 